\title{
Stability of a Time Discrete Perturbed Dynamical System with Delay
}

\author{
MICHAEL I. GIL ${ }^{\prime a *}$ and SUI SUN CHENG ${ }^{\mathrm{b}}$ \\ a Department of Mathematics, Ben Gurion University, P.O. Box 653, Beer Sheva 84105, Israel; \\ ${ }^{\mathrm{b}}$ Department of Mathematics, Tsing Hua University, Hsinchu, Taiwan 30043, ROC
}

(Received 11 February 1999)

Let $C^{n}$ be the set of $n$ complex vectors endowed with a norm $\|\cdot\|_{C^{n}}$. Let $A, B$ be two complex $n \times n$ matrices and $\tau$ a positive integer. In the present paper we consider the nonlinear difference equation with delay of the type

$$
u_{k+1}=A u_{k}+B u_{k-\tau}+F_{k}\left(u_{k}, u_{k-\tau}\right), \quad k=0,1,2, \ldots,
$$

where $F_{k}: C^{n} \times C^{n} \rightarrow C^{n}$ satisfies the condition

$$
\left\|F_{k}(x, y)\right\|_{C^{n}} \leq p\|x\|_{C^{n}}+q\|y\|_{C^{n}}, \quad k=0,1,2, \ldots,
$$

where $p$ and $q$ are positive constants. In this paper, absolute stability conditions for this equation are established.

Keywords: Nonlinear discrete system with delay, Absolute $l^{2}$-stability, Norm estimate

Let $C^{n}$ be the set of $n$ complex vectors endowed with a norm $\|\cdot\|_{C^{n}}$. Let $A, B$ be two complex $n \times n$ matrices and $\tau$ be a positive integer. Consider the perturbed difference equation with delay

$$
\begin{gathered}
u_{k+1}=A u_{k}+B u_{k-\tau}+F_{k}\left(u_{k}, u_{k-\tau}\right), \\
\quad k=0,1,2, \ldots,
\end{gathered}
$$

where $F_{k}: C^{n} \times C^{n} \rightarrow C^{n}$ satisfies the condition

$$
\begin{aligned}
& \left\|F_{k}(x, y)\right\|_{C^{n}} \leq p\|x\|_{C^{n}}+q\|y\|_{C^{n}}, \\
& \quad k=0,1,2, \ldots,
\end{aligned}
$$

where $p$ and $q$ are positive constants. In this paper, we will be concerned with the stability problem of Eq. (1) under the condition that all the zeroes of

$$
\operatorname{det}\left(z I-A-z^{-\tau} B\right)
$$

lie in the open unit disk in the complex plane $C^{1}$ with center at the origin, where $I$ stands for the identity matrix. In other words, we will assume that the greatest modulus $\rho(A, B ; \tau)$ of the roots of

\footnotetext{
* Corresponding author.
} 
$\operatorname{det}\left(A+z^{-\tau} B-z I\right)$ is less than 1. Similar problems have been dealt with perturbed equations of the form

$$
u_{k+1}=A u_{k}+f\left(x_{k}\right), \quad k=0,1,2, \ldots,
$$

as early as 1929 (see, e.g. Ortega, 1973), under the assumption that the spectral radius of $A$ is less than 1. For recent and related investigations, the readers may consult Ortega (1973), Xie and Cheng (1995, 1996) and others. We remark that in case $n=2$, our equation can be used to model the population growth of two species that are under delayed migration and interaction, see, e.g. Sandefur (1990, Chapter 7).

There are many concepts of stability for discrete time dynamical systems. Here we will adopt a specific one described as follows. First, note that a solution of (1) is a sequence $\left\{u_{k}\right\}_{k=-\tau}^{\infty}$ of vectors in $C^{n}$ such that it renders (1) into an identity after substitution. Given initial vectors $u_{-\tau}, u_{-\tau+1}, \ldots, u_{0}$, it is easily seen that we can successively calculate $u_{1}$, $u_{2}, \ldots$ according to (1) in a unique manner. An existence and uniqueness theorem for (1) can thus be easily formulated and proved. Next, note that the assumption (2) implies that the zero sequence $0=\{0\}_{k=-\tau}^{\infty}$ is a solution of (1). Let us take $l^{2}\left(C^{n}\right)$ to be the Hilbert space of all complex sequences of the form $v=\left\{v_{k}\right\}_{k=0}^{\infty}$ endowed with the usual inner product and norm

$$
\|v\|_{l^{2}}=\left\{\sum_{k=0}^{\infty}\left\|v_{k}\right\|_{C^{n}}^{2}\right\}^{1 / 2} .
$$

We say that the zero solution of (1) is absolutely $l^{2}$-stable (in the class of nonlinearities (2)) if there is a constant $\Gamma$ depending only on the numbers $p$ and $q$ such that for any solution $u=\left\{u_{k}\right\}_{k=-\tau}^{\infty}$, the truncated sequence $\tilde{u}=\left\{u_{k}\right\}_{k=0}^{\infty}$ satisfies

$$
\|\tilde{u}\|_{l^{2}} \leq \Gamma \sum_{k=-\tau}^{0}\left\|u_{k}\right\|_{C^{n}}
$$

We remark that it would have been better to replace the last inequality by

$$
\left\{\sum_{k=1}^{\infty}\left\|u_{k}\right\|_{C^{n}}^{2}\right\}^{1 / 2} \leq \tilde{\Gamma} \sum_{k=-\tau}^{0}\left\|u_{k}\right\|_{C^{n}}
$$

in the above definition, but clearly the two concepts are equivalent and we will keep the present definition for technical reasons (to be seen below).

Our main concern in this paper is to derive a stability criterion for the zero solution. To this end, let us first consider the following nonhomogeneous equation

$$
u_{k+1}=A u_{k}+B u_{k-\tau}+f_{k}, \quad k=0,1,2, \ldots
$$

Lemma 1 Suppose $\|f\|_{l^{2}}<\infty$ and $\rho(A, B ; \tau)<1$. Then the truncated sequence $\tilde{u}=\left\{u_{k}\right\}_{k=0}^{\infty}$ of a solution $\left\{u_{k}\right\}_{k=-\tau}^{\infty}$ of (3) will satisfy

$$
\|u\|_{l^{2}} \leq M\|f\|_{l^{2}}+\Gamma \sum_{k=-\tau}^{0}\left\|u_{k}\right\|_{C^{n}}
$$

for some constant $\Gamma$, where

$$
M=\max _{|z|=1}\left\|\left(z I-A-z^{-\tau} B\right)^{-1}\right\|_{C^{n}} .
$$

Proof First note that the sequence $\left\{f_{k}\right\}_{k=0}^{\infty}$ is of exponential order. Thus the solution $\left\{u_{k}\right\}_{k=-\tau}^{\infty}$ is also of exponential order (see e.g., Györi and Ladas, 1991, Lemma 1.4.2). Let $u:[-\tau, \infty) \rightarrow C^{n}$ and $f:[0, \infty) \rightarrow \infty$ be the piecewise continuous step functions defined by

$$
u(t)=u_{k}, \quad k \leq t<k+1, k=0,1,2, \ldots ;
$$

and

$$
f(t)=f_{k}, \quad k \leq t<k+1, k=0,1,2, \ldots
$$

Then the Laplace transforms of the restricted functions $\left.u\right|_{[0, \infty)}$ and $f$ exist. Let their Laplace transforms be denoted by $u^{*}$ and $f^{*}$ respectively, 
that is,

$$
u^{*}(z)=\int_{0}^{\infty} \mathrm{e}^{-z t} u(t) \mathrm{d} t
$$

and

$$
f^{*}(z)=\int_{0}^{\infty} \mathrm{e}^{-z t} f(t) \mathrm{d} t,
$$

where $z$ is the dual complex variable. In view of (3), we see that

$$
u(t+1)=A u(t)+B u(t-\tau)+f(t), \quad t \geq 0,
$$

thus multiplying both sides of the above equation by $\mathrm{e}^{-z t}$ and then integrating from 0 to $\infty$, we obtain

$$
\begin{aligned}
\mathrm{e}^{z}\left\{u^{*}(z)-\int_{0}^{1} \mathrm{e}^{-z t} u(t) \mathrm{d} t\right\} \\
=A u^{*}(z)+\mathrm{e}^{-\tau z} B\left\{u^{*}(z)+\int_{-\tau}^{0} \mathrm{e}^{-z t} u(t) \mathrm{d} t\right\} \\
\quad+f^{*}(z)
\end{aligned}
$$

or

$$
\begin{aligned}
& \left\{\mathrm{e}^{z} I-A-\mathrm{e}^{-\tau z} B\right\} u^{*}(z) \\
& =\left\{\mathrm{e}^{z} \int_{0}^{1} \mathrm{e}^{-z t} u(t) \mathrm{d} t+B \mathrm{e}^{-\tau z} \int_{-\tau}^{0} \mathrm{e}^{-z t} u(t) \mathrm{d} t\right. \\
& \left.\quad+f^{*}(z)\right\}
\end{aligned}
$$

Thus, in view of the assumption that $\rho(A, B ; \tau)<1$,

$$
\begin{aligned}
u^{*}(z)= & \left\{\mathrm{e}^{z} I-A-\mathrm{e}^{-\tau z} B\right\}^{-1} \\
& \times\left\{\mathrm{e}^{z} \int_{0}^{1} \mathrm{e}^{-z t} u(t) \mathrm{d} t\right. \\
& \left.\quad+B \mathrm{e}^{-\tau z} \int_{-1}^{0} \mathrm{e}^{-z t} u(t) \mathrm{d} t+f^{*}(z)\right\}
\end{aligned}
$$

for $z=\mathrm{i} \theta$ where $\theta \in R$. Note that

$$
\int_{0}^{1} \mathrm{e}^{-z t} u(t) \mathrm{d} t=\frac{1-\mathrm{e}^{-\tau z}}{z} u_{0}
$$

and

$$
\begin{aligned}
\int_{-\tau}^{0} \mathrm{e}^{-z t} u(t) \mathrm{d} t & =\sum_{k=-\tau}^{-1} \int_{k}^{k+1} u_{k} \mathrm{e}^{-z t} \mathrm{~d} t \\
& =\sum_{k=-\tau}^{-1} \frac{\mathrm{e}^{-z k}-\mathrm{e}^{-z(k+1)}}{z} u_{k}
\end{aligned}
$$

for $z=\mathrm{i} \theta$, and since for $k=-\tau,-\tau+1, \ldots,-1,0$

$$
\begin{aligned}
& \left|\int_{-\infty}^{\infty} \theta^{-1}\left(\mathrm{e}^{-\mathrm{i} \theta k}-\mathrm{e}^{-\mathrm{i} \theta(k+1)}\right) \mathrm{d} \theta\right|^{2} \\
& \quad \leq \int_{-\infty}^{\infty} \theta^{-2}\left|1-\mathrm{e}^{-\mathrm{i} \theta}\right|^{2} \mathrm{~d} \theta \\
& \quad=4 \int_{-\infty}^{\infty} \theta^{-2} \sin ^{2} \theta \mathrm{d} \theta \\
& \quad<\infty
\end{aligned}
$$

we see further that

$$
\begin{aligned}
& \left\{\int_{-\infty}^{\infty}\left\|\int_{-\tau}^{0} \mathrm{e}^{-\mathrm{i} \theta t} u(t) \mathrm{d} t\right\|_{C^{n}}^{2} \mathrm{~d} \theta\right\}^{1 / 2} \\
& \leq\left\{4 \int_{-\infty}^{\infty} \theta^{-2} \sin ^{2} \theta \mathrm{d} \theta\right\} \sum_{k=-\tau}^{-1}\left\|u_{k}\right\|_{C^{n}}
\end{aligned}
$$

and

$$
\begin{aligned}
& \left\{\int_{-\infty}^{\infty}\left\|\int_{0}^{1} \mathrm{e}^{-\mathrm{i} \theta t} u(t) \mathrm{d} t\right\|_{C^{n}}^{2} \mathrm{~d} \theta\right\}^{1 / 2} \\
& \leq\left\{4 \int_{-\infty}^{\infty} \theta^{-2} \sin ^{2} \theta \mathrm{d} \theta\right\}\left\|u_{0}\right\|_{C^{n}}
\end{aligned}
$$

In view of (5) and the above calculations, we now have

$$
\begin{aligned}
& \left\{\int_{-\infty}^{\infty}\left\|u^{*}(\mathrm{i} \theta)\right\|_{C^{n}}^{2} \mathrm{~d} \theta\right\}^{1 / 2} \\
& \quad \leq M\left\{\int_{-\infty}^{\infty}\left\|f^{*}(\mathrm{i} \theta)\right\|_{C^{n}}^{2} \mathrm{~d} \theta\right\}^{1 / 2}+\Gamma \sum_{k=-\tau}^{0}\left\|u_{k}\right\|_{C^{n}}
\end{aligned}
$$


for some constant $\Gamma$. Finally, from Parseval's equality, we have

$$
\begin{aligned}
& \left\{\int_{0}^{\infty}\|u(t)\|_{C^{n}}^{2} \mathrm{~d} t\right\}^{1 / 2} \\
& \quad \leq M\left\{\int_{0}^{\infty}\|f(t)\|_{C^{n}}^{2} \mathrm{~d} t\right\}^{1 / 2}+\Gamma \sum_{k=-\tau}^{0}\left\|u_{k}\right\|_{C^{n}}
\end{aligned}
$$

which implies

$$
\begin{aligned}
& \left\{\sum_{k=0}^{\infty}\left\|u_{k}\right\|_{C^{n}}^{2}\right\}^{1 / 2} \\
& \quad \leq M\left\{\sum_{k=0}^{\infty}\left\|f_{k}\right\|_{C^{n}}^{2}\right\}^{1 / 2}+\Gamma \sum_{k=-\tau}^{0}\left\|u_{k}\right\|_{C^{n}},
\end{aligned}
$$

as required. The proof is complete.

THEOREM 1 Suppose $\rho(A, B ; \tau)<1 \quad$ and $(p+q) M<1$, where $M$ is defined by (4). Then the zero solution of (1) is absolutely $l^{2}$-stable.

Proof Let $\left\{u_{k}\right\}_{k=-\tau}^{\infty}$ be a solution of (1). For each nonnegative integer $m$, let the sequence $h^{(m)}=$ $\left\{h_{k}^{(m)}\right\}_{k=0}^{\infty}$ be defined by $h_{k}^{(m)}=1$ for $0 \leq k \leq m$ and $h_{k}^{(m)}=0$ for $k>m$, and let $v^{(m)}=\left\{v_{k}^{(m)}\right\}_{k=-\tau}^{\infty}$ be the unique solution determined by the conditions

$$
v_{k}^{(m)}=u_{k}^{(m)}, \quad k=-\tau,-\tau+1, \ldots, 0
$$

and

$$
\begin{gathered}
v_{k+1}^{(m)}=A v_{k}^{(m)}+B v_{k-\tau}^{(m)}+h_{k}^{(m)} F_{k}\left(v_{k}^{(m)}, v_{k-\tau}^{(m)}\right), \\
k=0,1,2, \ldots
\end{gathered}
$$

Clearly, by uniqueness,

$$
v_{k}^{(m)}=u_{k}^{(m)}, \quad-\tau \leq k \leq m+1 .
$$

Furthermore, if we let $f^{(m)}=\left\{f_{k}^{(m)}\right\}_{k=0}^{\infty}$ be defined by

$$
f_{k}^{(m)}=h_{k}^{(m)} F_{k}\left(v_{k}^{(m)}, v_{k-\tau}^{(m)}\right), \quad k \geq 0,
$$

then since

$$
\begin{aligned}
\left\|f^{(m)}\right\|_{l^{2}} & =\left\{\sum_{k=0}^{m}\left\|F_{k}\left(u_{k}^{(m)}, u_{k-\tau}^{(m)}\right)\right\|_{C^{n}}^{2}\right\}^{1 / 2} \\
\leq & (p+q)\left\{\sum_{k=0}^{m}\left\|u_{k}^{(m)}\right\|_{C^{n}}^{2}\right\}^{1 / 2} \\
& +q\left\{\sum_{k=-\tau}^{0}\left\|u_{k}\right\|_{C^{n}}^{2}\right\}^{1 / 2}<\infty
\end{aligned}
$$

thus by Lemma 1, we see that

$$
\begin{gathered}
\left\{\sum_{k=0}^{m}\left\|u_{k}^{(m)}\right\|_{C^{n}}^{2}\right\}^{1 / 2} \\
\leq\left\|v^{(m)}\right\|_{l^{2}} \\
\leq M(p+q)\left\{\sum_{k=0}^{m}\left\|u_{k}^{(m)}\right\|_{C^{n}}^{2}\right\}^{1 / 2} \\
+(M q+\Gamma)\left\{\sum_{k=-\tau}^{0}\left\|u_{k}\right\|_{C^{n}}^{2}\right\}^{1 / 2}
\end{gathered}
$$

or

$$
\begin{aligned}
& \left\{\sum_{k=0}^{m}\left\|u_{k}^{(m)}\right\|_{C^{n}}^{2}\right\}^{1 / 2} \\
& \leq \frac{M q+\Gamma}{1-M(p+q)}\left\{\sum_{k=-\tau}^{0}\left\|u_{k}\right\|_{C^{n}}^{2}\right\}^{1 / 2} .
\end{aligned}
$$

Finally, letting $m$ tend to $\infty$, we see that our desired result holds. The proof is complete.

The constant $M$ in the above Theorem is stated in terms of the inverse matrix of $z I-A-z^{-\tau} B$. This is inconvenient in general. In order to obtain a more convenient estimate, we proceed as follows. Let us first take $\|\cdot\|_{C^{n}}$ to denote the Euclidean norm in the sequel. Further, let $\lambda_{1}(H), \ldots, \lambda_{n}(H)$ be the eigenvalues of an $n \times n$ complex matrix $H$ including their multiplicities. We will make use of the following 
quantity:

$$
g(H)=\left\{N^{2}(H)-\sum_{k=1}^{n} \lambda_{k}(H)\right\}^{1 / 2},
$$

where $N(H)$ is the Frobenius (Hilbert-Schmidt) norm of $H$, i.e.

$$
N^{2}(H)=\operatorname{Trace}\left(H H^{*}\right) .
$$

There are a number of properties of $g(H)$ which are useful, see Gil' (1995, Section 1.2). Here we note that

$$
\begin{aligned}
& g(H) \leq \sqrt{\frac{1}{2}} N\left(H^{*}-H\right), \\
& g\left(H \mathrm{e}^{\mathrm{i} \xi}+z I\right)=g(H), \quad \xi \in R, z \in C,
\end{aligned}
$$

and that if $H$ is normal, i.e. $H H^{*}=H^{*} H$, then $g(H)=0$. Furthermore, for some particular matrices, the corresponding $g$ can be easily calculated. For example, if $H=\left(h_{i j}\right)$ is a triangular matrix such that $h_{i j}=0$ for $1 \leq j<i \leq n$, then

$$
g^{2}(H)=\sum_{1 \leq i<j \leq n}\left|h_{i j}\right|^{2} .
$$

To facilitate descriptions, we will adopt the convention that $0 !=1,0^{0}=1$ and empty sums are zero. We will also make use of the following notations. The binomial coefficient $C_{j}^{i}$ is given by

$$
C_{j}^{i}=\frac{i !}{j !(i-j) !}, \quad 0 \leq j \leq i,
$$

as usual, but we will also adopt the convention that $C_{j}^{i}=0$ when $j<0$ or $j>i$. We will define

$$
\gamma_{n, i}=\sqrt{\frac{C_{i}^{n-1}}{(n-1)^{i}}}, \quad i=0,1,2, \ldots, n-1,
$$

and $\gamma_{n, i}=0$ if $i<0$ or $i>n-1$. Note that $\gamma_{n, 0}=1$ and

$$
\gamma_{n, i}^{2}=\frac{(n-2)(n-3) \cdots(n-i)}{(n-1)^{i-1} i !} \leq \frac{1}{i !} .
$$

Finally, we let $\rho_{0}\left(A+z^{-\tau} B-z I\right)$ be the smallest modulus of the eigenvalues of the matrix $A+$ $z^{-\tau} B-z I$, and let

$$
\Lambda_{0}=\max _{|z|=1} \sum_{k=0}^{n-1} \frac{\gamma_{n, k} g^{k}\left(A+z^{-\tau} B\right)}{\rho_{0}^{k+1}\left(A+z^{-\tau} B-z I\right)} .
$$

The quantities $g\left(A+z^{-\tau} B\right), \rho_{0}\left(A+z^{-\tau} B-z I\right)$ and hence $\Lambda_{0}$ are more manageable than the quantity $M$. Indeed, in view of (8) and (9),

$$
\begin{aligned}
g(A & \left.+z^{-\tau} B\right) \\
& \leq \sqrt{\frac{1}{2}}\left\{N\left(A^{*}-A\right)+N\left(\bar{z}^{-\tau} B^{*}-z^{-\tau} B\right)\right\} \\
& \leq \sqrt{\frac{1}{2}}\left\{N\left(A^{*}-A\right)+2|z|^{-\tau} N(B)\right\},
\end{aligned}
$$

so that

$$
\begin{aligned}
\Lambda_{0} \leq & \sum_{k=0}^{n-1} \frac{\gamma_{n, k}}{\rho_{0}^{k+1}\left(A+z^{-\tau} B-z I\right)} \\
& \times\left\{\frac{N\left(A^{*}-A\right)+2 N(B)}{\sqrt{2}}\right\}^{k} \equiv \Lambda_{1} .
\end{aligned}
$$

We may also show that

$$
M \leq \Lambda_{0} .
$$

In fact, if $H$ is a linear operator on $C^{n}$, then the following estimate is true (see Gil', 1995, p. 5):

$$
\left\|R_{\lambda}(H)\right\|_{C^{n}} \leq \sum_{k=0}^{n-1} g^{k}(H) \frac{\gamma_{n, k}}{d^{k+1}(H, \lambda)}, \quad \lambda \notin \sigma(H),
$$

where $d(H, \lambda)$ is the distance between the spectrum $\sigma(H)$ of $H$ and the complex number $\lambda$. Hence for any invertible matrix $H$,

$$
\left\|H^{-1}\right\|_{C^{n}} \leq \sum_{k=0}^{n-1} g^{k}(H) \frac{\gamma_{n, k}}{\rho_{0}^{k+1}(H)},
$$


where $\rho_{0}(H)$ is the smallest modulus of the has roots eigenvalues of $H$. Relation (12) yields

$$
\begin{aligned}
& \left\|\left(A+z^{-\tau} B-z I\right)^{-1}\right\|_{C^{n}} \\
& \quad \leq \sum_{k=0}^{n-1} g^{k}\left(A+z^{-\tau} B-z I\right) \frac{\gamma_{n, k}}{\rho_{0}^{k+1}\left(A+z^{-\tau} B-z I\right)}
\end{aligned}
$$

for regular $z$, so that, in view of (9),

$$
\begin{aligned}
M & =\max _{|z|=1}\left\|\left(A+z^{-\tau} B-z I\right)^{-1}\right\|_{C^{n}} \\
& \leq \max _{|z|=1} \sum_{k=0}^{n-1} \frac{\gamma_{n, k} g^{k}\left(A+z^{-\tau} B-z I\right)}{\rho_{0}^{k+1}\left(A+z^{-\tau} B-z I\right)} \\
& =\max _{|z|=1} \sum_{k=0}^{n-1} \frac{g^{k}\left(A+z^{-\tau} B\right) \gamma_{n, k}}{\rho_{0}^{k+1}\left(A+z^{-\tau} B-z I\right)}=\Lambda_{0},
\end{aligned}
$$

as desired.

As a consequence, we see that if $\rho(A, B ; \tau)<1$, and $(p+q) \Lambda_{0}<1$ or $(p+q) \Lambda_{1}<1$, where $\Lambda_{0}$ and $\Lambda_{1}$ are defined by (10) and (11) respectively, then the zero solution of (1) is absolutely $l^{2}$-stable.

We now consider a specific example to illustrate our previous results. Let $\tau=1, A$ be the $2 \times 2$ identity matrix and $B$ be the diffusion matrix with real parameter $\mu$ :

$$
B=\mu\left(\begin{array}{cc}
2 & -1 \\
-1 & 2
\end{array}\right)
$$

Then the "characteristic matrix" $z I-A-z^{-1} B$ is equal to

$$
\frac{1}{z}\left(\begin{array}{cc}
z^{2}-z-2 \mu & \mu \\
\mu & z^{2}-z-2 \mu
\end{array}\right)
$$

which has eigenvalues

$$
\frac{1}{z^{2}}\left(z^{3}-z^{2}-z \mu\right), \quad \frac{1}{z^{2}}\left(z^{3}-z^{2}-3 z \mu\right),
$$

and its determinant

$$
\operatorname{det}\left(A+z^{-1} B-z I\right)=\frac{1}{z^{2}}\left\{\left(z+2 \mu-z^{2}\right)^{2}-\mu^{2}\right\}
$$

Thus for $-1 / 12 \leq \mu<0$,

$$
\begin{aligned}
& \rho(A, B ; \tau) \\
& \quad=\max \left\{\left|\frac{1}{2} \pm \frac{1}{2} \sqrt{1+12 \mu}\right|,\left|\frac{1}{2} \pm \sqrt{1+4 \mu}\right|\right\} \in(0,1)
\end{aligned}
$$

and

$$
\begin{aligned}
& \rho_{0}\left(A+z^{-\tau} B-z I\right) \\
& \quad=\min _{|z|=1} \min \{|(1-\mu) z-1|,|(1-3 \mu) z-1|\} .
\end{aligned}
$$

For instance, when $\mu=-0.05$,

$$
\begin{aligned}
& \rho_{0}\left(A+z^{-\tau} B-z I\right) \\
& \quad=\min _{|z|=1} \min \{|1.05 z-1|,|1.15 z-1|\} \\
& =0.0025 .
\end{aligned}
$$

Furthermore, since the matrix $I+z^{-\tau} B$ is normal, we have $g\left(I+z^{-\tau} B\right)=0$. Thus, if $-1 / 12 \leq \mu<0$ and

$$
\begin{aligned}
p+q & \leq \Lambda_{0} \\
& =\frac{1}{\min _{|z|=1} \min \{|(1-\mu) z-1|,|(1-3 \mu) z-1|\}},
\end{aligned}
$$

then the two interacting species model

$$
\begin{gathered}
u_{k+1}-u_{k}=\mu\left(\begin{array}{cc}
2 & -1 \\
-1 & 2
\end{array}\right) u_{k-1}+F_{k}\left(u_{k}, u_{k-1}\right), \\
k=0,1,2, \ldots
\end{gathered}
$$

is absolutely $l^{2}$-stable.

\section{References}

Driver, R.D., Ladas, G. and Vlahos, P.N. (1992). Asymptotic behavior of a linear delay difference equation, Proc. Amer. Math. Soc., 115, 105-112.

Gil', M.I. (1995). Norm Estimations for Operator-Valued Functions and Applications, Marcel Dekker, Inc., New York.

Györi, I. and Ladas, G. (1991). Oscillation Theory of Delay differential Equations, Clarendon Press, Oxford. 
Ortega, J.M. (1973). Stability of difference equations and convergence of iterative processes, SIAM J. Numer. Anal., 10(2), 268-282.

Sandefur, J.T. (1990). Discrete Dynamical Systems, Clarendon Press, Oxford.

Xie, S.L. and Cheng, S.S. (1995). Decaying solutions of neutral difference equations with delays, Ann. of Diff. Eqs., 11(3), $331-345$.

Xie, S.L. and Cheng, S.S. (1996). Stability criteria for parabolic type partial difference equations, J. Comp. Appl. Math., 75, $57-66$. 


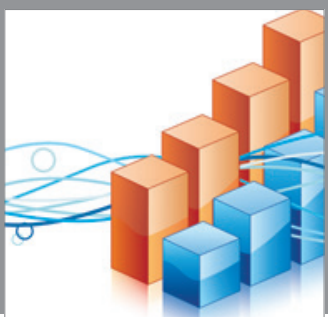

Advances in

Operations Research

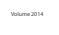

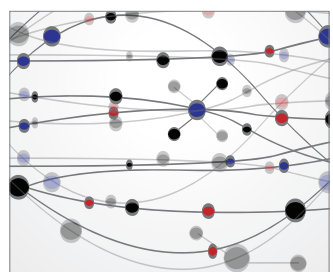

\section{The Scientific} World Journal
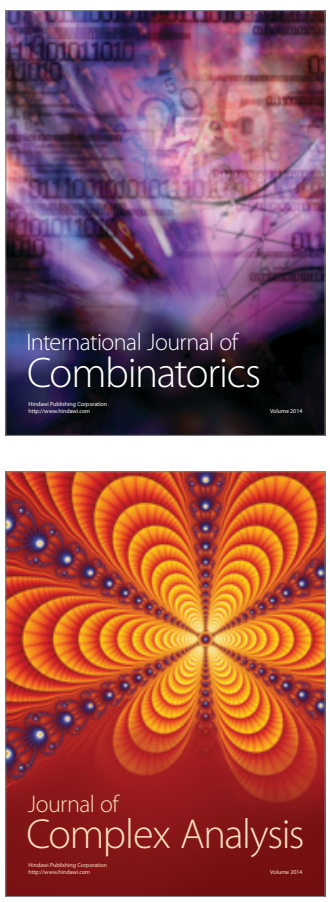

International Journal of

Mathematics and

Mathematical

Sciences
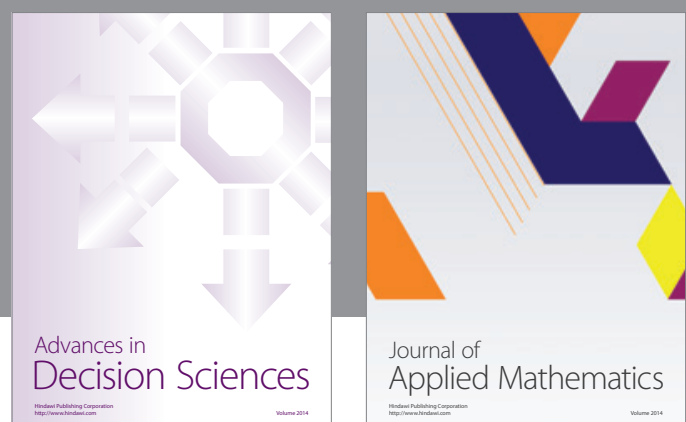

Journal of

Applied Mathematics
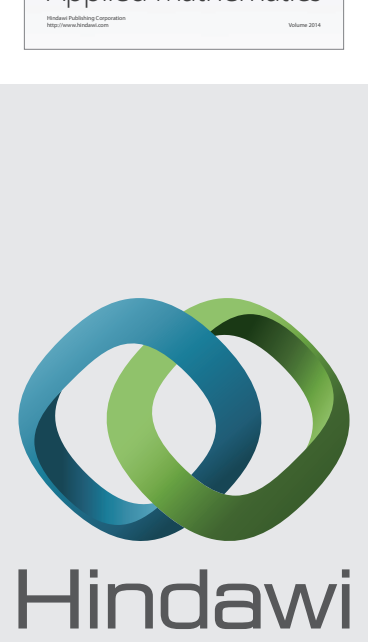

Submit your manuscripts at http://www.hindawi.com
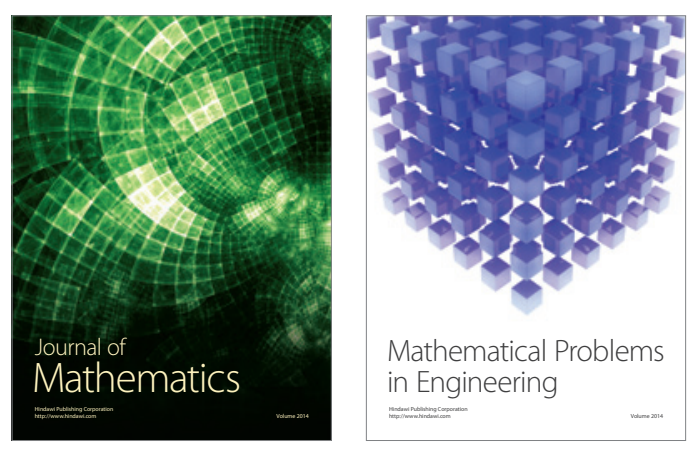

Mathematical Problems in Engineering
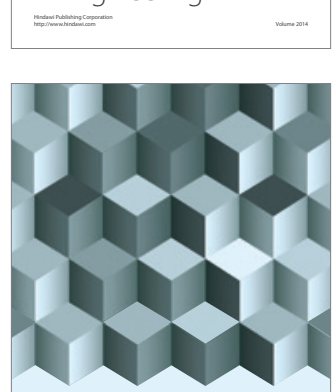

Journal of

Function Spaces
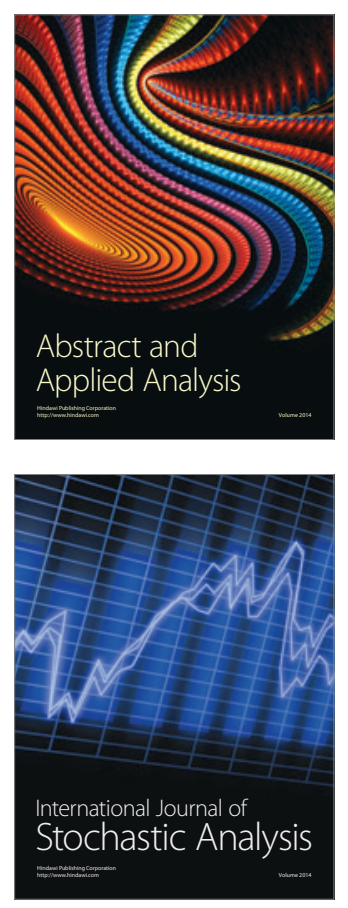

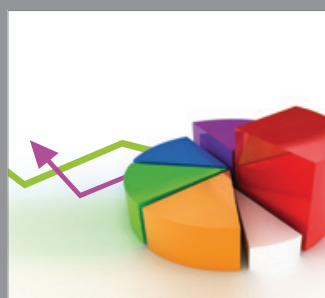

ournal of

Probability and Statistics

Promensencen
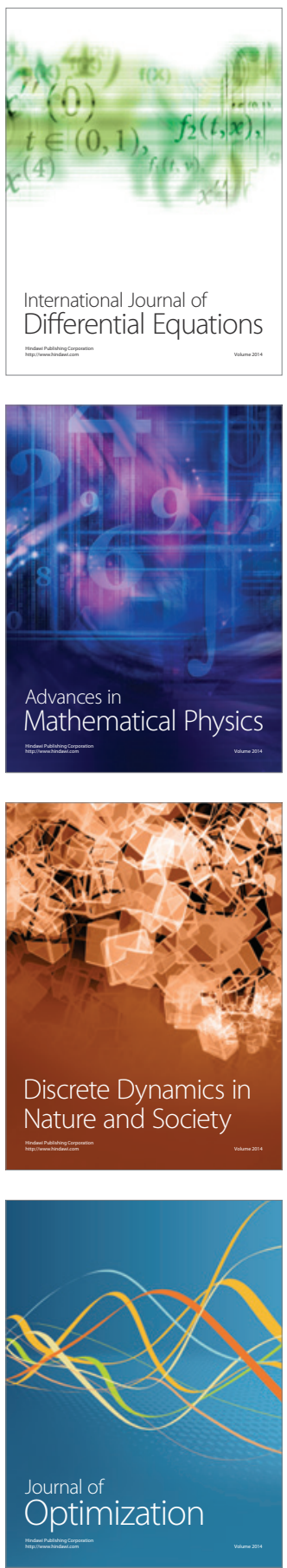